Working

Paper

Department

of Economics

$\mathrm{Ca}^{\prime}$ Foscari University of

Venice

Sergio Currarini

Group Stability of

Hierarchies in Games

with Spillovers 


\title{
Group Stability of Hierarchies in Games with Spillovers
}

\author{
Sergio Currarini \\ University of Venice
}

\begin{abstract}
In a recent paper, Demange (2004) has shown that hierarchical organizations can guarantee the existence of stable cooperative outcomes by appropriately allocating the blocking power to a subset of coalitions, the "teams". This paper extends the analysis of Demange to cooperative problems with spillovers. We show that if blocking coalitions have "pessimistic expectations" on the reaction of outsiders, in all cooperative problems there exists an allocation which is blocked by no team. We also study the case of "passive expectations", for which the same result holds in all games with negative spillovers, while stable allocations may fail to exist in games with positive spillovers. In the latter class of games, however, hierarchies are shown to be the most stable organizational forms.
\end{abstract}

\section{Keywords}

Hierarchies, Coalitions, Stability, Spillovers, Cooperative Games, Networks.

\section{JEL Codes}

C7, C71

\author{
Address for correspondence: \\ Sergio Currarini \\ Department of Economics \\ Ca' Foscari University of Venice \\ Cannaregio 873, Fondamenta S.Giobbe \\ 30121 Venezia - Italy \\ Phone: (++39) 0412349133 \\ Fax: (++39) 0412349176 \\ e-mail: s.currarini@unive.it
}

This Working Paper (o QD se in inglese) is published under the auspices of the Department of Economics of the Ca' Foscari University of Venice. Opinions expressed herein are those of the authors and not those of the Department. The Working Paper series (o QD se in inglese) is designed to divulge preliminary or incomplete work, circulated to favour discussion and comments. Citation of this paper should consider its provisional character. 


\section{Introduction}

Formal economic organizations arise as "means to achieve the benefits of collective action in situations in which the price system fails" (Arrow, (1974)). Among the possible forms of organization, hierarchies are by far the most widespread in economic, social and political life. Formally, a hierarchy can be represented as a pyramidal network: a "principal" is at the top of the organization, and each organizational member has a unique direct superior. The chain of authority is such that each other organizational member has a unique path of intermediate superiors through which he can communicate with the principal.

In a recent paper, Demange (2004) has proposed new and insightful elements for a theoretical explanation of the emergence of hierarchies. Differently from most works on the subject, which focus on the design of efficient organizational forms ${ }^{1}$, Demange (2004) studies the effect of a hierarchical order on the stability of cooperation. Taking a core-theoretic approach to the problem, Demange focuses on the constraints that the hierarchy imposes on the ability of subsets of players to organize in coalitions and to object to (or to "block") a collective decision taken by the organization. ${ }^{2}$ By forcing players to report to a common superior, the hierarchy endows only special coalitions - the "teams" - to act autonomously of the rest of the organization. Teams have the desired property of containing a player which is superior in the hierarchy to all other team members and, as such, can coordinate their actions. Demange shows that to all cooperative problems we can associate a collective decision, called "hierarchical outcome", which is blocked by no team in the hierarchy.

In Demange (2004), the payoff possibilities of each team are entirely determined by the actions taken by its members. If utility is transferable, the cooperative situations studied by Demange can be represented as games in characteristic function. Many important instances of cooperative decision making do not, however, satisfy this assumption. On the contrary, it is often the case that the payoff possibilities of a coalition depend also on the actions taken by the players outside the coalition. These "spillovers" arise in many economic and political cooperative situations where hierarchies are a widespread forms of organization and stability is a relevant issue.

\footnotetext{
${ }^{1}$ See the early contribution of Coase (1937), and, more receintly, Williamson (1975) and the subsequent extensive literature, surveyed in Milgrom and Roberts (1992).

${ }^{2} \mathrm{~A}$ prior investigation of group stability when only a subclass of coalitions are effective in raising objections (the "basic" coalitions) is contained in Kaneko and Wooders (1982) and, for a very simple form of hierarchy, in Greenberg and Weber (1983).
} 
In vertically integrated industries, for instance, major companies may have an incentive to divest some of their production units to independent companies. The case of AT\&T, giving up its equipment division, and General Motors, giving up its auto part division, are two eminent recent examples of an intrinsic instability of the vertically integrated structure. Within the boundaries of the firm, another main source of instability is the incentives of employees to make use of the training obtained within the firm to start their own independent business, often in competition with their former employers. The case of Silicon Valley (and, in general, of the high-tech industry) is a documented example where engineers and other professionals often change firms or quit their jobs to start firms of their own, bringing with them projects and licences. When an engineer involves in his new independent project his own close collaborators, such defection take the form of "team blocking" in the sense made clear above. Political competition is still another example in which relevant spillovers from coalition formation coexist with alliances which are internally organized as trees (think, for instance, of the order of parties' ideological positions on the left-right arrow). ${ }^{3}$

This paper extends Demange (2004) analysis to include such cooperative situation with spillovers. In order to maintain the stability analysis based on the primitive notion of "coalitional blocking", specific assumptions on the expected reaction of outside players need to be introduced, where by reaction we mean the configuration of outside players in coalitions after the blocking. A formulation of reactions which are endogenously determined by the strategic interaction among coalitions has motivated several recent papers in the coalition theory literature (see, among others, Ray and Vohra (1997)). In this paper we do not attempt such a task, by rely on exogenous assumption about players expectations.

We first study the case in which blocking coalitions form "pessimistic expectations", in the sense that they consider the maximal payoff they can guarantee to their members independently of the organization of outsiders. The use of these guarantee levels dates back to Von Neumann and Morgenstern's (1944) analysis of games in normal form and has recently been adapted by Funaki and Yamato (1999) to problems of coalition formation. We show in

\footnotetext{
${ }^{3}$ Although political alliance may not be endowed with a clear hierarchical order, still teams can be identified with coalitions which are consecutive in the ideological order. In fact Demange's results can be reformulated by replacing the notion of a team (based on the hierarchical order) by the notion of an "internally connected" coalition (based on the architecture of the network defining the internal organization of a coalition). In the absence of a hierarchical order, the restriction of blocking power to connected coalitions is justified in terms of communication and coordination possibilities.
} 
Theorem 1 that Demange's result fully extends: whenever agents face gains from cooperation, the hierarchical outcome is blocked by no team, independently of the shape of spillovers. We establish this result also for the case in which the "punishment" of blocking coalitions is limited by the constraints imposed by the hierarchy on the coordination possibilities of outsiders. ${ }^{4}$

We then turn to a less extreme form of expectations, with blocking coalitions expecting to get the maximal feasible payoff compatible with the prediction that outsiders will maintain the same bilateral relations that shape the hierarchical structure, and cooperate according to such relations. In other words, the hierarchy is taken as given by agents, and fully determine both their ability to coordinate on blocking and their ability to cooperate after blocking has taken place (in Ichiishi's (1997) terminology, these "passive expectations" underlie the notion of Strong Nash Equilibrium in normal form games).

With respect to the case of pessimistic expectations, this case is characterized by more profitable coalitional objections, and the stability result of Demange (2004) does not extend in general. However, we show in Proposition 2 that all hierarchies are stable in all problems with negative spillovers. ${ }^{5}$ Although no stable allocation may exist under positive spillovers ${ }^{6}$, Proposition 2 shows that hierarchies remain the most stable organizational forms in the following sense: all allocations that are stable for some non hierarchical organization must be stable for some hierarchical organization as well.

The paper is organized as follows. Section 2 presents the concepts and notation. Section 3 presents the main results. Section 4 concludes the paper.

\section{Preliminaries}

\subsection{Games in Partition Function Form}

We consider a set $N=\{1,2, \ldots, n\}$ of agents, called the grand coalition, with generic member $i \in N$. A coalition is any subset $S$ of $N$. For all $S \subseteq N$, we denote by $\Pi_{S}$ the set of all partitions $\pi_{S}$ of the set $S$, that is, all collections $\left\{B_{1}, B_{2}, \ldots, B_{m}\right\}$ of subsets of $S$ with empty

\footnotetext{
${ }^{4}$ These constraints are the same that justify the restriction of blocking power to teams.

${ }^{5}$ Negative spillovers arise when the formation of a coalition (or the merger of more coalitions) hurt the other players in the system.(as, for instance, in custom unions and cost reducing R\&D alliances).

${ }^{6}$ Positive spillovers arise when the formation of a coalition benefits other players, as in public good games and Cournot oligopoly.
} 
pairwise intersection and whose union coincides with $S$. A partition $\pi_{S} \in \Pi_{S}$ is also called a coalition structure on $S$.

Letting $\pi \in \Pi_{N}$ and $S \in \pi$, we call the pair $(S, \pi)$ an embedded coalition. A partition function maps each embedded coalition $(S, \pi)$ into a real number $v(S, \pi)$, denoting the aggregate payoff generated by coalition $S$ when embedded in partition $\pi$. With slight abuse of notation, we will write $v(\pi)$ for $\sum_{B \in \pi} v(B, \pi)$. Given an embedded coalition $(S, \pi)$, we denote by $\pi \backslash S$ the partition of the set $N \backslash S$ obtained by considering all the elements of $\pi$ except $S$.

A partition function accounts for the fact that a coalition's payoff possibilities may depend on the configuration of other coalitions. Two classes of partition functions have played a central role in the literature, and cover many well known cooperative problems. These classes refer to the sign of the welfare effect exerted on a coalition $S$ by a "concentration" of the coalition structure adopted by players in $N \backslash S$. The notion of concentration is defined as follows: ${ }^{7}$

Definition 1 Let $S \subseteq N$. The partition $\pi \in \Pi_{S}$ is a concentration of $\pi^{\prime} \in \Pi_{S}$ if $\pi \neq \pi^{\prime}$ and if it is possible to originate $\pi$ by merging elements of $\pi^{\prime}$. Formally, $\pi$ is a concentration of $\pi^{\prime}$ if for all $B \in \pi$ either $B \in \pi^{\prime}$ or there exists a collection $\left\{B_{1}, . ., B_{p}\right\}$ of elements of $\pi^{\prime}$ such that $B=\bigcup_{k=1}^{p} B_{k}$.

Definition 2 The partition function $v$ exhibits positive spillovers if $v(S, \pi) \geq v\left(S, \pi^{\prime}\right)$ whenever $\pi$ is a concentration of $\pi^{\prime}$.

Definition 3 The partition function $v$ exhibits negative spillovers if $v(S, \pi) \leq v\left(S, \pi^{\prime}\right)$ whenever $\pi$ is a concentration of $\pi^{\prime}$.

A partition function is cohesive when the grand coalition generates more value than any other coalition structure.

Definition 4 The function $v$ is cohesive if for all partitions $\pi \in \Pi_{N}$ :

$$
v(N,\{N\}) \geq v(\pi) .
$$

\footnotetext{
${ }^{7}$ The definition given by Yi (1997) and used in other works in the literature (see for instance the surveys by Bloch $(1997,2003)$ ), makes use of the notion of concentration of a partition, and is more general than the one used here.
} 
Full cohesiveness is a stronger condition, requiring that each coalition generates a higher value than any of its partitions, for any coalition structure in which it may be embedded. In defining this property, and elsewhere in the paper, we will use the superadditive cover $\hat{v}$ of $v$, defined as follows for any $\pi_{S} \in \Pi_{S}$ and $\pi_{N \backslash S} \in \Pi_{N \backslash S}$ :

$$
\hat{v}\left(S,\left\{\pi_{S}, \pi_{N \backslash S}\right\}\right) \equiv \sum_{B \in \pi_{S}} v\left(B,\left\{\pi_{S}, \pi_{N \backslash S}\right\}\right) .
$$

Definition 5 The function $v$ is fully cohesive if for all embedded coalitions $(S, \pi)$ and all $\bar{\pi}_{S} \in \Pi_{S}:$

$$
v(S, \pi) \geq \hat{v}\left(S,\left\{\bar{\pi}_{S}, \pi \backslash S\right\}\right) .
$$

The different requirements imposed by cohesiveness and full cohesiveness deserve further discussion. Cohesiveness basically requires that coordination of individual strategies is always (weakly) better than the absence of cooperation. For instance, it requires that a monopolistic cartel generates more aggregate profit than any other market structure. It would be indeed difficult to argue that the members of the cartel are not allowed to replicate the same strategies they would play in any other market structure. Full cohesiveness, in contrast, may fail because of the interplay between different coalitions. So, two Cournot triopolist may generate a higher (Nash Equilibrium) aggregate profit than if they merged to form a duopoly. Although after merging they would be able to replicate the equilibrium strategy they played as triopolists, these strategies may fail to be part of the equilibrium of the duopoly game, from which the failure of full cohesiveness.

\subsection{Graphs and Hierarchies}

We now introduce some basic concepts, borrowed from the theory of graphs, that will allow us to formally represent the hierarchical organizations adopted by the grand coalition $N$.

\subsubsection{Graphs}

Given a set of vertices $N$, a graph (or network) $g$ on $N$ is defined as any subset of the set of all (unordered) pairs of vertices in $N$ :

$$
g \subseteq\{i j: i \in N \text { and } j \in N, i \neq j\} .
$$

The pair $i j \in g$ is called a "link", and the link $i j$ is called "incident" to vertices $i$ and $j$. The set of vertices that are incident to at least one link in the graph $g$ is denoted by $N(g)$. 
The graph $g$ is connected if for all pairs of vertices $i$ and $j$ in $N$ there exists a connecting path $P(i, j)$, that is, a set of vertices $\left\{i_{1}, i_{2}, \ldots, i_{k}\right\}$ such that $i=i_{1}, j=i_{k}$, and $i_{p} i_{p+1} \in g$ for all $p=1, \ldots, k-1$. A coalition $S \subset N$ is connected in $g$ if for all pairs of vertices $i$ and $j$ in $S$ there exists a connecting path $P(i, j)$ in $g$ all included in $S$. The set of connected coalitions in $g$ is denoted by $C C(g)$. Given the graph $g$ with vertex set $N$, the graph $h$ with vertex set $S \subseteq N$ is a subgraph of $g$ if $h \subseteq g$. The subgraph $h$ of $g$ is a component of $g$ if it is connected and if for all $i \in N(h)$ and $j \notin N(h)$ we have $i j \notin g$.

\subsubsection{Connected graphs as organizations}

If we interpret each player in the players' set $N$ as a vertex, the connected graph $g$ on $N$ can be viewed as the organizational form through which the grandcoalition achieves coordination. As suggested by Myerson (1977), the graph can be thought of as describing the communication possibilities of players in $N$, directly implying that the set of players $N$ acts as a "coalition", in the sense of cooperative game theory, if and only if $g$ is connected. More generally, the set $C(g)$ of components of a disconnected graph $g$ describes a set of coexisting organizations. We can express the coalitions of cooperating players in a graph $g$ through the associated partition $\pi(g)$, uniquely obtained as follows:

$$
\pi(g)=\{N(h): h \in C(g)\}
$$

It will be sometimes useful to refer to the restriction $\left.g\right|_{S}$ of a graph $g$ to the subset of vertices $S \subset N$; the restricted graph $\left.g\right|_{S}$ has vertex set $S$ and contains all the links (and only those) of $g$ that are not incident to vertices in $N \backslash S$.

\subsubsection{Trees and hierarchies}

The graph $g$ contains a cycle if there exists a path $P(i, j)$ in $g$ that contains at least three vertices and for which $i=j$. A connected graph containing no cycle is called a tree (or minimally connected graph). The tree $g$ is a "spanning tree" of the connected graph $g^{\prime}$ if $N(g)=N\left(g^{\prime}\right)$ and $g \subset g^{\prime}$.

It is possible to interpret trees as hierarchies by selecting a vertex $r$ and defining the partial order $\succ_{g, r}$ on $N$ as follows:

$$
j \succ_{g, r} k \Longleftrightarrow k \in P(r, j)
$$


Since $r \succ_{g, r} k$ for all $k \in N, r$ is the top element of the order $\succ_{g, r}$. We say that $r$ is the root of the hierarchy. For all other vertices $j$ an $k$ we read $j \succ_{g, r} k$ as " $j$ follows $k$ in $g$ with root $r "$.

Definition 6 A hierarchy is a pair $(g, r)$, where $g$ is a tree defined on the set of vertices $N$ and $r \in N$.

We say that vertex $k$ is a direct superior of vertex $j$ if $j \succ_{g, r} k$ and $j k \in g$. We will denote by $F_{g}(i)$ the set of all vertices that follow vertex $i$ in $g$, including vertex $i$ itself (see figure 1 below). For each $S \subset N$, we denote by $D_{g}(S)$ the set of direct followers of $S$ in $g$, that is, those vertices that are not in $S$ but whose direct superior belongs to $S$. Finally, we denote by $T_{g}$ the set of terminal vertices in the hierarchy $g$, that is, those vertices with no followers.

It is clear that every connected coalition $S$ in a tree $g$ defines a restricted graph $\left.g\right|_{S}$ which is itself a tree. Once we have specifies a root $r$ for $g$, then every connected coalition $S$ defines a new hierarchy $\left(\left.g\right|_{S}, i(S)\right)$, where $i(S)$ is the uniquely defined vertex in $S$ which is not followed by any vertex in $S$. Following Demange (2004), we will refer to such connected coalitions as teams (see Figure 1 below). Note that the set of teams of a hierarchy is independent of the choice of the root $r$. For this reason we will refer to the set of teams of the tree $g$.

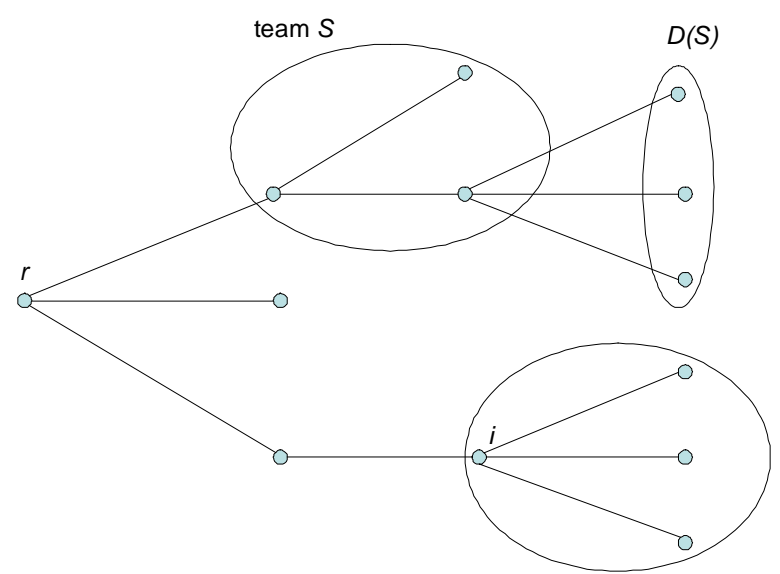

$F(i)$

Figure 1: A Hierarchy 


\subsection{Feasible and Efficient Allocations}

A payoff allocation for the grand coalition $N$ is a vector $u \in R_{+}^{n}$ of individual payoffs for the members of $N$. The allocation $u$ is feasible under partition function $v$ is there exists a way in which the players in $N$ can partition themselves and generate the values distributed by $u$.

Definition 7 The payoff allocation $u \in R_{+}^{n}$ is feasible under partition function $v$ if there exists a coalition structure $\pi \in \Pi_{N}$ such that $\sum_{i \in B} u_{i} \leq v(B, \pi)$ for all $B \in \pi$. In this case we say that $\pi$ supports $u$.

Note that the organizational form(s) adopted by players does not enter the above definition. Having defined payoff possibilities in terms of a partition function we have implicitly assumed that the type of hierarchical structure adopted by players does not affect the aggregate payoff they can generate by cooperating.

Among feasible allocations we will focus on those which are efficient in the sense of the following definition:

Definition 8 The feasible payoff allocation $u \in R_{+}^{n}$ is efficient under partition function $v$ if there exists no other feasible allocation $u^{\prime}$ for which $u_{i}^{\prime}>u_{i}$ for all $i \in N$.

If the function $v$ is cohesive, then the grand coalition supports all efficient allocations.

\section{Stability}

In this section we establish a set of results that extend the stability features of hierarchies proved in Demange (2004) to games in partition function.

\subsection{Games Without Spillovers: Demange's Result}

The analysis of Demange (2004) takes as primitive the claims that each coalition $S$ has on aggregate payoffs. Such claims are measured by the feasible allocations that the members of $S$ can guarantee themselves by acting without the cooperation of the players in $N \backslash S$. In Demange (2004), these allocations are fully determined by the set of actions available to the members of $S$. In a context of transferable utility, these claims can be expressed in terms of a a "characteristic function" $\phi$, mapping each coalition $S \subseteq N$ into a real number $\phi(S)$. The 
function $\phi$ is a special case of a partition function $v$ for which $v(S, \pi)=v\left(S, \pi^{\prime}\right)$ for all $\pi$ and $\pi^{\prime}$.

If any pair of disjoint coalitions increase their claims by acting together, the function $\phi$ is said to be superadditive.

Definition 9 The characteristic function $\phi$ is superadditive if for all $S \subset N$ and $T \subset N$ such that $S \cap T=\emptyset$ the following inequality holds:

$$
\phi(S)+\phi(T) \leq \phi(S \cup T) .
$$

Claims define the incentives of each coalition to "block" any proposed payoff allocation for the grand coalition.

Definition 10 Let $\phi$ be superadditive. The payoff allocation $u$ is blocked by coalition $S \subseteq N$ if $\sum_{i \in S} u_{i}<\phi(S)$.

When all coalitions have the "ability" to block, the set of unblocked allocations defines the core of the associated cooperative game $(N, \phi)$.

The main point raised by Demange (2004) is that the hierarchical structure (and, more in general, any internal organization adopted by the grand coalition) may limit the blocking ability of certain coalitions by limiting the possibility that their members communicate without the help of players outside the coalition. In Demange's paper, this ability is assigned only to the set of "teams" in the hierarchy (see section 2.2.3), in which a common superior coordinate the joint blocking decision. Demange (2004) has shown that a specific allocation, called the "hierarchical outcome", distributes the efficient aggregate payoff in such a way that the claims of all teams are satisfied. When the characteristic function $\phi$ is superadditive, the hierarchical outcome $u \in R_{+}^{n}$ associated with the hierarchy $(g, r)$ is obtained as follows: each terminal player $i \in T_{g}$ is allocated the payoff $u_{i}=\phi(\{i\})$. Any player $i \notin T_{g}$ is allocated the difference between $\phi\left(F_{g}(i)\right)$ and the sum $\sum_{i \in F_{g}(i) \backslash\{i\}} u_{i}$.

Theorem 1 (Theorem 1 in Demange (2004)). Let $\phi$ be superadditive. The hierarchical outcome associated with the hierarchy $(g, r)$ is not blocked by any team in $g$.

Theorem 2 in Demange's paper studies the case of non superadditive decision problems. It is there shown that a variation of the hierarchical outcome described above is not blocked 
by any team and is supported by some partition of the set of players $N$. We discuss this possibility after proposition 1 in the next section.

\subsection{Games with Spillovers: Pessimistic Expectations}

When a hierarchical organization operates in the presence of spillovers, the claim of each blocking coalition does not depend solely on the actions available to its members, but also on the actions that the other players in the organization are expected to take in case of blocking. Specific assumptions are needed on the coalition structure that would be induced as a consequence of each coalitional blocking.

In this section we study the case of "pessimistic expectations", first suggested by von Neumann and Morgenstern (1944) for games in normal form and recently applied to the analysis of games in partition function form by Funaki and Yamato (1999). ${ }^{8}$ The spirit of pessimistic expectations is to assume that each blocking coalition considers as its claim the maximal payoff it can guarantee to its members, independently of the reaction of outsiders. Formally, the claim of coalition $S$ under partition function $v$ is defined by the following auxiliary characteristic function:

$$
\phi(S) \equiv \max _{\pi_{S} \in \Pi_{S}} \min _{\pi_{N \backslash S} \in \Pi_{N \backslash S}} \hat{v}\left(S,\left\{\pi_{S}, \pi_{N \backslash S}\right\}\right)
$$

Remark 1 Definition (2) implicitly says that even if $S$ decides to split in the coalition structure $\pi_{S}$, the players in $S$ are still able to commit to any sort of transfers, independently of the subcoalition they end up belonging to in $\pi_{S}$. This assumption obviously gives stronger incentives to block than if such transfers were not allowed. However, since the results of propositions 1 and 2 will prove the existence of unblocked allocations, the same result would a fortiori hold if transfers were not allowed.

Remark 2 Pessimistic expectations take a clear-cut form in the classes of games with negative and positive spillovers. In the first class, players in $N \backslash S$ are expected to stay together in one united coalition; in the second, these players are expected to split up into the singletons partition of the set $N \backslash S$. We will return to these two classes of games and on the role of expectations in the section 3.3.

\footnotetext{
${ }^{8}$ See also Hart and Kurz (1983), defining the $\alpha$-core and the $\beta$-core of games of coalition formation.
} 
Remark 3 Both the members of $S$ and of $N \backslash S$ are allowed to partition themselves in any arbitrary way, independently of the starting hierarchical structure $g$. In certain applications, where the constraint on coordination imposed by the graph $g$ survive after the blocking of $S$, this assumption may not be appropriate, and both the optimal choice of $S$ and the reaction of $N \backslash S$ should be subject to the existing coordination possibilities.

The definition of the function $\phi$ can be modified in order to account for the observations raised in remark 3. For any tree $g$ and any coalition $C \subset N$, we denote by $\Pi_{C}^{g}$ the set of partitions $\pi_{C} \in \Pi_{C}$ that are also subpartitions of $\pi\left(\left.g\right|_{C}\right)$ (the partition derived from $g$ by considering the set of components of the restricted graph $\left.g\right|_{C}$ ). So, every element of $\pi_{C}$ is either an element or a subset of some element of $\pi\left(\left.g\right|_{C}\right)$. The claim of a blocking coalition $S$ in $g$ is redefined as follows:

$$
\phi_{g}(S) \equiv \max _{\pi_{S} \in \Pi_{S}^{g}} \min _{\pi_{N \backslash S} \in \Pi_{N \backslash S}^{g}} \hat{v}\left(S,\left\{\pi_{S}, \pi_{N \backslash S}\right\}\right)
$$

Note that we cannot say whether a coalition $S$ is made better by blocking under characteristic functions $\phi$ or under $\phi_{g}$. In fact, while the first allows for more effective "punishments" by $N \backslash S$, the second restricts the set of possibilities of $S$.

The following proposition extends theorem 1 to all games in partition function form, for both the associated characteristic functions $\phi$ and $\phi_{g}$.

Proposition 1 The characteristic functions $\phi$ and $\phi_{g}$ are superadditive for all partition functions $v$.

Proof. We prove the result for the function $\phi$. All steps are valid for function $\phi_{g}$ as well. For any set $C \subset N$, define the function $f_{C}: \Pi_{C} \rightarrow \Pi_{N \backslash C}$ as follows: ${ }^{9}$

$$
f_{C}\left(\pi_{C}\right)=\arg \min _{\pi_{N \backslash C} \in \Pi_{N \backslash C}} \hat{v}\left(C,\left\{\pi_{C}, \pi_{N \backslash C}\right\}\right) .
$$

Consider now any pair of disjoint coalitions $S, T$. We need to show that

$$
\phi(S)+\phi(T) \leq \phi(S \cup T) .
$$

\footnotetext{
${ }^{9}$ For simplicity, we are restricting the analysis for simplicity to partition functions $v$ such that $v\left(C,\left\{\pi_{C}, \pi_{N \backslash C}\right\} \neq v\left(C,\left\{\pi_{C}, \bar{\pi}_{N \backslash C}\right\}\right.\right.$ whenever $\pi_{N \backslash C} \neq \bar{\pi}_{N \backslash C}$. This assumption can be easily dropped at the cost of a lenghtier proof.
} 
Definition (2) applied to $S \cup T$ gives:

$$
\phi(S \cup T)=\max _{\pi_{(S \cup T)} \in \Pi_{(S \cup T)}} \hat{v}\left(S \cup T,\left\{\pi_{S \cup T}, f_{S \cup T}\left(\pi_{S \cup T}\right)\right\}\right) .
$$

We can therefore write:

$$
\phi(S \cup T) \geq \max _{\left(\pi_{S}, \pi_{T}\right) \in \Pi_{S} \times \Pi_{T}} \hat{v}\left(S \cup T,\left\{\pi_{S}, \pi_{T}, f\left(\pi_{S}, \pi_{T}\right)\right\}\right) .
$$

Consider now the values $\phi(S)$ and $\phi(T)$. Denote by $\hat{\pi}_{S}$ and $\hat{\pi}_{T}$ the partitions of $S$ and $T$ that originate such values:

$$
\begin{aligned}
& \hat{\pi}_{S}=\arg \max _{\pi_{S} \in \Pi_{S}} \hat{v}\left(S,\left\{\pi_{S}, f_{S}\left(\pi_{S}\right)\right\}\right) \\
& \hat{\pi}_{T}=\arg \max _{\pi_{T} \in \Pi_{T}} \hat{v}\left(T,\left\{\pi_{T}, f_{T}\left(\pi_{T}\right)\right\}\right) .
\end{aligned}
$$

By definition of the functions $\phi$ and $f_{S \cup T}$, the following inequalities hold:

$$
\begin{aligned}
& \phi(S) \leq \hat{v}\left(S,\left\{\hat{\pi}_{S}, \hat{\pi}_{T}, f_{S \cup T}\left(\hat{\pi}_{S}, \hat{\pi}_{T}\right)\right\}\right) \\
& \phi(T) \leq \hat{v}\left(T,\left\{\hat{\pi}_{S}, \hat{\pi}_{T}, f_{S \cup T}\left(\hat{\pi}_{S}, \hat{\pi}_{T}\right)\right\}\right) . .
\end{aligned}
$$

Inequalities (7) and (9) directly imply:

$$
\begin{gathered}
\phi(S \cup T) \geq \max _{\left(\pi_{S}, \pi_{T}\right) \in \Pi_{S} \times \Pi_{T}} \hat{v}\left(S \cup T,\left\{\pi_{S}, \pi_{T}, f\left(\pi_{S}, \pi_{T}\right)\right\}\right) \geq \\
\geq \hat{v}\left(S,\left\{\hat{\pi}_{S}, \hat{\pi}_{T}, f_{S \cup T}\left(\hat{\pi}_{S}, \hat{\pi}_{T}\right)\right\}\right)+\hat{v}\left(T,\left\{\hat{\pi}_{S}, \hat{\pi}_{T}, f_{S \cup T}\left(\hat{\pi}_{S}, \hat{\pi}_{T}\right)\right\}\right) \geq \phi(S)+\phi(T) .
\end{gathered}
$$

The fact that functions $\phi$ and $\phi_{g}$ are superadditive does not, without further restrictions on $v$, ensure that the hierarchical outcomes associated with such functions define feasible payoff imputations in the sense of definition 7 . In fact, if $v$ is not cohesive, then the partitions $\pi^{*}$ and $\pi_{g}^{*}$ that generate the values $\phi(N)$ and $\phi_{g}(N)$ may contain more than one set. Definitions 7 would require therefore that the hierarchical outcomes satisfy the requirement that each coalition $B \in \pi^{*}$ (resp., $B \in \pi_{g}^{*}$ ) receives exactly an aggregate payoff of $v\left(B, \pi^{*}\right)$ (resp., $v\left(B, \pi_{g}^{*}\right)$. However, as shown in the next example, this is not true in general because of the effect of spillovers.

Example 1 Consider the hierarchy $g=\{12,23\}$, with root 1 . Let the partition function $v$ be such that:

$$
\begin{aligned}
\pi_{g}^{*} & =\{12,3\} \\
v(3,\{12,3\}) & >v(3,\{1,2,3\}) .
\end{aligned}
$$


In this case the hierarchical outcome $u$ for $\phi_{g}$ allocates to 3 less than the value generated by 3 in $\pi_{g}^{*}$, violating definition 7 :

$$
u_{3}=\phi_{g}(3)=v(3,\{1,2,3\})<v\left(3, \pi_{g}^{*}\right)
$$

This problem is due to the very definitions of $\phi(N)$ and of $\phi_{g}(N)$, which implicitly assume that transfers across elements of $\pi^{*}$ and $\pi_{g}^{*}$ are possible, while such transfers are not allowed by definition 7 . For this reason, in the next theorem we need to impose that $v$ is cohesive, thereby ensuring that no transfers across coalitions are needed at the hierarchical outcome. We remark, however, that cohesiveness of $v$ does not require a superadditivity property for all coalitions (as, in contrast, would be required by full cohesiveness), but only that maximal gains from trade are exploited by the grand coalition.

Theorem 2 Let $g$ be a tree with set of vertex $N$, and let $v$ be a cohesive partition function. For all $r \in N$, if coalitional claims are measured by the characteristic function $\phi$ (resp., by $\left.\phi_{g}\right)$, the hierarchical outcome associated with the hierarchy $(g, r)$ and with $\phi$ (resp., with $\phi_{g}$ ) is a feasible payoff allocation supported by the grandcoalition and is blocked by no team in $g$.

Proof. Cohesiveness of $v$ implies that $\{N\} \in \arg \max _{\pi \in \Pi_{N}} v(\pi)$. Superadditivity of the functions $\phi$ and $\phi_{g}$ imply that the hierarchical outcome allocates a non negative payoff to all players and that it exactly exhausts the value $v(N,\{N\})$. The fact that hierarchical outcomes are not blocked by any team in $g$ is directly implied by Proposition 1 and Theorem 1 .

Note, finally, that transfers across coalitions are implicit in the definitions of $\phi(S)$ and of $\phi_{g}(S)$ for any coalition $S \subset N$. Although such transfers imply a violation of definition 7 for the reasons made clear above, the overstatement of the blocking power of coalitions that derives from this violation reinforces the stability result of theorem 1.

We conclude this section by applying the above analysis to three simple examples of vertical integration, political competition and international cooperation. While the first application is derived in full detail, the other two are briefly sketched.

Example 2 Vertical Integration - Consider three vertically integrated firms, $A, B$ and $R$. Firms $A$ and $B$ jointly produce a consumption good to be sold on the market by firm $R$ (the retailer). Firm $A$ buys inputs on a competitive factor market at constant marginal cost $c$, and produces an intermediate good $x_{A}$ that firm $B$ uses as input. Firm $B$ transforms each 
unit of good $x_{A}$ into one unit of final good. Firm $R$ finally markets the final good. The produced goods are specific to the integrated firms, in the sense that there is no alternative market for these goods outside the vertical structure. The vertically integrated structure acts as a monopolist and makes the monopolist profit $u_{M}$. A payoff allocation for the vertically integrated firm is any distribution of $u_{M}$ among the three firms. Blocking is here identified with the decision to opt out of the vertically integrated structure and to act independently of what remains of it. For instance, the retailer may opt out by deciding to buy the final good from $B$, to market the good and to receive the profits. Also, firms $A$ and $B$ may jointly opt out of the vertical structure and sell their output to firm $R$. Similarly, firms $B$ and $R$ can opt out, buy the intermediate input from firm $A$ and sell the final good on the market. However, firms $A$ and $R$ cannot exert the same threat, since they cannot jointly produce anything without the contribution of firm $B$.

We derive the partition function for this problem by looking at the equilibrium profits in the price setting game in which firms move sequentially according to the vertical structure $A, B, R$. For all proper partitions of $N$, we will assume that upstream firms set their prices first, and that the demand for the final good is given by $1-p_{R}$. We obtain the following values.

\begin{tabular}{||l||l||l||}
\hline \hline$\{A B R\}$ & $p_{M}=\frac{1+c}{2}$ & $v(N) \equiv \frac{(1-c)^{2}}{4}$ \\
\hline \hline \multirow{2}{*}{$A, B R$} & $p_{A}=\frac{1+c}{2}$ & $v(A,\{A, B R\}) \equiv \frac{(1-c)^{2}}{8}$ \\
& $p_{B R}=\frac{3+c}{4}$ & $v(B R,\{A, B R\}) \equiv \frac{(1-c)^{2}}{16}$ \\
\hline \hline \multirow{2}{*}{$A B, R$} & $p_{A B}=\frac{1+c}{2}$ & $v(A B,\{A B, R\}) \equiv \frac{(1-c)^{2}}{8}$ \\
& $p_{R}=\frac{3+c}{4}$ & $v(R,\{A B, R\}) \equiv \frac{(1-c)^{2}}{16}$ \\
\hline \hline \multirow{4}{*}{$A, B, R$} & $p_{A}=\frac{1+c}{2}$ & $v(A,\{A, B, R\}) \equiv \frac{(1-c)^{2}}{16}$ \\
& $p_{B}=\frac{3+c}{4}$ & $v(B,\{A, B, R\}) \equiv \frac{(1-c)^{2}}{32}$ \\
& $p_{R}=\frac{7+c}{8}$ & $v(R,\{A, B, R\}) \equiv \frac{(1-c)^{2}}{64}$ \\
\hline \hline
\end{tabular}

We then derive function $\phi_{g}$ for this problem. Expectations do not play any role in determining the claims of coalitions $A B, B R$ and $B$. Pessimistic expectations are instead used to derive the claims of firms $A$ and $R$ : we obtain $\phi(A)=\frac{(1-c)^{2}}{16}$ and $\phi(R)=\frac{(1-c)^{2}}{64}$. Summing up we get:

\begin{tabular}{||l||l||l||c||c||}
\hline \hline$\phi_{g}(A B)$ & $\phi_{g}(B R)$ & $\phi_{g}(B)$ & $\phi_{g}(A)$ & $\phi_{g}(R)$ \\
\hline \hline$\frac{(1-c)^{2}}{8}$ & $\frac{(1-c)^{2}}{16}$ & $\frac{(1-c)^{2}}{32}$ & $\frac{(1-c)^{2}}{16}$ & $\frac{(1-c)^{2}}{64}$ \\
\hline \hline
\end{tabular}


The function $\phi_{g}$ is superadditive, and theorem 2 directly implies that the hierarchical outcome is not blocked by any connected coalition of firms. To appreciate the role of the hierarchy in generating stable allocations, consider what would happen if disconnected coalitions could block. To obtain the claim of the disconnected coalition $A R$ we cannot simply vertically integrate these two firms, since this is not technologically feasible by assumption. We could however allow $A$ and $R$ to collude on prices. So, firm $R$ sets its price given the prices set by $A$ and $B$; firm $B$ sets $p_{B}$ given $p_{A}$; firm $A$ sets $p_{A}$ so as to maximize the aggregate profit of $A$ and $R$, given the optimal reactions of $B$ and $R$. Simple computations give us the following prices and profits: $p_{A}=\frac{2 c+1}{3}, p_{B}=\frac{2+c}{3}, p_{R}=\frac{5+c}{6} ; v(A R,\{A R, B\})=\frac{(1-c)^{2}}{12}$. Note that although the function $\phi_{g}$ is superadditive, the core of the game $\left(N, \phi_{g}\right)$ is empty. In fact, the core constraints for coalitions $A B, B R$ and $A R$ imply that

$$
u_{A}+u_{B}+u_{R} \geq \frac{(1-c)^{2}}{4} \frac{13}{12}>v(A B R,\{A B R\}) .
$$

Example 3 Political Alliances. - Consider a political alliance in which parties $L, M$ and $R$ are ordered on a one dimensional spectrum of ideological positions. The alliance has to take decisions on various issues, on which parties may have different opinions. If a party or a subset of parties is not happy with a proposed decision, it can threaten to defect from the alliance. However, while a restricted alliance between parties $L$ and $M$ could be envisaged, an alliance between $L$ and $R$ may not credible without the mediation of $M$. This situation can be modeled by assuming that there is some fixed utility $\bar{u}$ from being in power, from which each party $i$ has to subtract the square of the distance between the chosen policy $x$ and its preferred policy $x_{i}$. If we assume that $\bar{u}$ is small enough we obtain that the aggregate utility of coalition $(L R)$ is always negative, while the aggregate value of the three party alliance and of every other two party alliance is strictly positive. Under these circumstances, exiting the alliance is likely to be less attractive for $R$ than for $M$, the former having to face a larger and more powerful competitor. The game has therefore negative spillovers.

Example 4 International Cooperation: At the international level, bilateral relations between countries are characterized by different degrees of trust and friendship. An agreement to increase domestic production of an international public good (e.g., abatement of polluting emissions) is possible only if cooperating countries are connected in the "trust" network. This 
constraint applies both to the global cooperation process (involving the grand coalition) and to possible objections (involving subcoalitions of participants proposing alternative partial agreements or inaction). Suppose that each country $i$ has a utility function $q+x_{i}$, defined on the aggregate internationally produced public good $q$ and a private good consumption $x_{i}$. The public good is produced by each country with strictly convex cost function $c\left(q_{i}\right)$, where $q_{i}$ is the production of public good of country $i$, and $q \equiv \sum_{i} q_{i}$ (all countries are therefore symmetric). Given some initial endowment of private good, in the absence of cooperation each country chooses $q_{i}$ to maximize the function $q-c\left(q_{i}\right)$ with respect to $q_{i}$. If a given partition $\pi$ emerges, each element $B$ of $\pi$ acts as a single player, maximizing the aggregate utility of the set of countries in $B$. Suppose there is one country $j$ that all countries trust and which trusts all countries, but no other links are present in the trust network (which has therefore a "star" architecture, with central vertex $j$ ). The game has positive spillovers, and the worst coalition structure for a blocking coalition $S$ is the one in which all countries in $N \backslash S$ split up into singletons. Moreover, the game is cohesive. Therefore, the stable hierarchical outcome associated with the hierarchy that has $j$ as root vertex allocates to all countries but $j$ their utility level at the non cooperative equilibrium, while country $j$ obtains all the gains from cooperation. In all other hierarchies (that is, for all other choices of the root), country $j$ still gets some gain from cooperation by virtue of its central position (by which it is never a terminal vertex of the network).

\subsection{Games with Spillovers: Passive Expectations}

Although under pessimistic expectations coalitional claims have the natural interpretation of "guarantee levels", other more optimistic behavioural principles can be envisaged. Players may think, for instance, that the links within the organization will stay on for some time after the objection. This may be a good assumption when the hierarchy is made of exogenous and fixed communication or technological constraints.

Such "passive expectations" (see Ichiishi (1997)) generate a characteristic function $\phi_{g}^{*}$ which differs from $\phi_{g}$ in that residual players do not sever existing links in order to "punish" the blocking coalition. It follows that claims are higher under $\phi_{g}^{*}$ than under $\phi_{g}$. The characteristic function $\phi_{g}^{*}$ is derived by looking at the value generated by the blocking coalition $S$ in the following partition:

$$
\pi(S, g) \equiv\left\{\pi\left(\left.g\right|_{S}\right), \pi\left(\left.g\right|_{N \backslash S}\right)\right\}
$$


The next example shows that stable allocation may fail to exist in this case:

Example 5 Let $N=\{1,2,3\}$, let $g=\{12,13\}$ and the (anonymous) function $v$ be as follows:

$$
\begin{aligned}
v(N,\{N\}) & =3 ; \\
\phi_{g}^{*}(2) & =v(2,\{2,13\})=2 ; \\
v(1,\{1,2,3\}) & =1-\varepsilon>0 ; \\
v(\{13\},\{13,2\}) & \equiv \phi_{g}^{*}(13)=2,
\end{aligned}
$$

with $\varepsilon>0$. The function $v$ satisfies positive spillovers. Considering the disjoint coalitions (1) and (23), we obtain:

$$
\phi_{g}^{*}(2)+\phi_{g}^{*}(13)=4>3=v(N,\{N\}),
$$

showing that $\phi_{g}^{*}$ is not superadditive. Also, it can be checked that no allocation exists that meet the claims of both player 2 and of coalition 13

In this example, the lack of stable allocation is generated by the strong incentives to free ride, due to the presence of positive spillovers. In such games, the harshest punishment for the blocking coalition is the disintegration of the residual organization, which is here prevented by the existing links, and is, in contrast, allowed under $\phi$ and $\phi_{g}$.

The same problem does not arise under negative spillovers. In fact, the structure $\pi\left(\left.g\right|_{N \backslash S}\right)$ induced by the coalition $S$ is the coarsest structure allowed to the players in $N \backslash S$ by the definition of $\phi_{g}$, and therefore the harshest punishment for $S$. The same result derived for the function $\phi_{g}$ for all partition functions extends therefore here for the class of functions with negative spillovers. This is stated as point i) of the next proposition. In point ii) we establish a property for games with positive spillovers, reaffirming the role of hierarchies for stability even in this class of games.

Proposition 2 i) Let $v$ be cohesive and exhibit negative spillovers and let $g$ be a tree. For all hierarchies $(g, r)$ the associated hierarchical outcome is blocked by no team. ii) Let $v$ be fully cohesive and exhibit positive spillovers. For all organizations $g^{\prime}$ containing cycles, if the allocation $\bar{u}$ is blocked by no team in $g^{\prime}$, then $u$ is blocked by no team in all the spanning trees of $g^{\prime}$. 
Proof. i) Proposition 1 has shown that no team in $g$ blocks the hierarchical outcome when $\phi_{g}$ is the characteristic function. Letting $u$ denote the hierarchical outcome, this means that for all teams $S \subset N$ we have:

$$
\phi_{g}(S)=\max _{\pi_{S} \in \Pi_{S}^{g}} \min _{\pi_{N \backslash S} \in \Pi_{N \backslash S}^{g}} \hat{v}\left(S,\left\{\pi_{S}, \pi_{N \backslash S}\right\}\right) \leq \sum_{i \in S} u_{i} .
$$

Since $\pi_{N \backslash S} \in \Pi_{N \backslash S}^{g}$ implies that $\pi_{N \backslash S}$ is a subpartition of $\pi\left(\left.g\right|_{N \backslash S}\right)$, given the negative spillovers of $v$ we can write for all teams $S$ :

$$
\phi_{g}^{*}(S) \leq \max _{\pi_{S} \in \Pi_{S}^{g}} \min _{\pi_{N \backslash S} \in \Pi_{N \backslash S}^{g}} \hat{v}\left(S,\left\{\pi_{S}, \pi_{N \backslash S}\right\}\right) \leq \sum_{i \in S} u_{i}
$$

ii) Consider any non hierarchical organization $g^{\prime}$, which, by definition, is not minimally connected (that is, contains cycles). Suppose that $g^{\prime}$ admits a stable allocation when coalitional claims are given by $\phi_{g}^{*}$. This means that for each connected coalition $S \in C C\left(g^{\prime}\right)$ we have $\sum_{i \in S} u_{i} \geq \phi_{g}^{*}(S)$. Consider now any spanning tree $g$ of $g^{\prime}$. Clearly, we have $C C(g) \subseteq C C\left(g^{\prime}\right)$. This means that all coalitions that have the ability to object to $g$ can also object to $g^{\prime}$. Also, by the full cohesiveness assumption, we need not worry about any partition $\pi_{S}$, since $S$ will always stay united. Since for each $S \in C C(g)$ each component of $\left.g^{\prime}\right|_{N \backslash S}$ is either a component of $\left.g\right|_{N \backslash S}$ or can be obtained by merging two or more components of $\left.g\right|_{N \backslash S}$, it follows that the partition $\pi\left(S, g^{\prime}\right)$ must concentrate the partition $\pi(S, g)$. Positive spillovers imply that $\phi_{g}^{*}(S) \leq \phi_{g^{\prime}}^{*}(S)$. We conclude that the stability of $g^{\prime}$ directly implies the stability of $g$.

Point ii) in Proposition 2 shows that, under positive spillovers, the set of allocations which are stable for at least one hierarchical structure includes all allocations which are stable for at least one arbitrary organization. This property does not extend to games with negative spillovers both under the functions $\phi_{g}$ and $\phi_{g}^{*}$. The reason is that, under these functions, adding links to a hierarchy (thereby creating cycles) has two effects: it enlarges the set of connected coalitions and it induces (weakly) more concentrated coalition structure after all objections. While both effects decrease the stability of the organization under positive spillovers (from which the result of proposition 2), they move in opposite directions under negative spillovers, where a larger set of connected coalitions possess weaker incentives to object. In this latter case, non hierarchical organizations may well sustain stable allocations which are objected in all hierarchies (including those generated by the spanning trees). 


\section{Conclusions}

We have studied the stability of collective decisions when players are organized in a hierarchy and coalitional payoff possibilities are described by a partition function. Our analysis has built on the work of Demange (2004), with which it shares the basic assumption that the hierarchy limits the ability to block collective decisions to the set of coalitions that are internally connected (the set of "teams"). We have shown that if blocking coalitions have "pessimistic expectations" about the reaction of residual players, then Demange's stability result carry over to all games in partition function form. If players form "passive expectations", then a stable allocation exists in all games with negative spillovers, but may fail to exist if spillovers are positive. In the latter case, however, hierarchies are still the most stable organizational form, since the set of allocations that are stable within a hierarchy include all allocations that are stable in at least one arbitrary organizational form.

\section{References}

[1] Arrow, K. J. (1974) The Limits of Organization, W. W. Norton, New York, London.

[2] Bloch, F. (1997) "Non Cooperative Models of Coalition Formation in Games with Spillovers," in Carraro C. and Siniscalco D. (eds.) New Directions in the Economic Theory of the Environment Cambridge University Press, Cambridge.

[3] Bloch, F. (2003) "Theories of Coalition Formation," in C. Carraro (ed) The endogenous formation of economic coalitions Fondazione Enrico Mattei Series in Economics and the Environment, Elgar, Cheltenham, U.K. and Northampton, Mass.

[4] Coase, R. (1937) "The Nature of the Firm", Economica, 4, 386-405.

[5] Demange, G. (2004) "On Group Stability in Hierarchies and Networks," Journal of Political Economy 112(4), 754 -7788.

[6] Funaki, Y., and Y. Yamato (1999) "The Core of an Economy with a Common Pool Resource: a Partition Function Approach," International Journal of Game Theory 28, $157-171$. 
[7] Greenberg, J. and S. Weber (1993) "Stable Coalition Structures in Consecutive Games," in Binmore, K., Kirman, A. and P. Tani (eds.) Frontiers of Games Theory, MIT Press, Cambridge and London, 103-115.

[8] Hart, S. and M. Kurz (1983) "Endogenous Formation of Coalitions," Econometrica 51, 1047-1064.

[9] Ichiishi, T. (1997) Microeconomi Theory, Blackwell Publishers, Oxford, U.K.

[10] Kaneko, M. and M.H. Wooders (1982) "Cores of Partitioning Games," Mathematical Social Sciences 3, 313-327.

[11] Milgrom, P., and J. Roberts (1992) Economics, Organization and Management Englewood Cliffs, Prentice-Hall.

[12] Myerson, R. (1977) "Graphs and Cooperation in Games," Math.Oper. Res. 2, 225-229.

[13] Radner, R. (1993) "The Organization of Decentralized Information Processing," Econometrica 61, 1009-1046.

[14] Ray, D., Vohra, R. (1997), "Equilibrium Binding Agreements", Journal of Economic Theory 73, 30-78.

[15] von Neumann, J., and O. Morgenstern (1944) Theory of Games and Economic Behaviour Princeton University Press, Princeton, New Jersey.

[16] Williamson, O. (1975) Markets and Hierarchies Free Press.

[17] Yi, S. S. (1997) "Stable Coalition Structures with Externalities," Games and Economic Behaviour 20, 201-237. 